International Journal of Neural Systems, Vol. 22, No. 2 (2012) 00310 (18 pages)

(C) World Scientific Publishing Company

DOI: $10.1142 /$ S0129065712003109

\title{
A NEURAL-VISUALIZATION IDS FOR HONEYNET DATA
}

\author{
ÁLVARO HERRERO \\ Department of Civil Engineering, \\ University of Burgos, Burgos, Spain \\ URKO ZURUTUZA \\ Electronics and Computing Department, \\ Mondragon University, Arrasate-Mondragon, Spain \\ EMILIO CORCHADO \\ Departamento de Informática y Automática, \\ Universidad de Salamanca, Salamanca, Spain \\ Department of Computer Science \\ Vঙ̆B-Technical University of Ostrava, Ostrava, Czech Republic
}

\begin{abstract}
Neural intelligent systems can provide a visualization of the network traffic for security staff, in order to reduce the widely known high false-positive rate associated with misuse-based Intrusion Detection Systems (IDSs). Unlike previous work, this study proposes an unsupervised neural models that generate an intuitive visualization of the captured traffic, rather than network statistics. These snapshots of network events are immensely useful for security personnel that monitor network behavior. The system is based on the use of different neural projection and unsupervised methods for the visual inspection of honeypot data, and may be seen as a complementary network security tool that sheds light on internal data structures through visual inspection of the traffic itself. Furthermore, it is intended to facilitate verification and assessment of Snort performance (a well-known and widely-used misuse-based IDS), through the visualization of attack patterns. Empirical verification and comparison of the proposed projection methods are performed in a real domain, where two different case studies are defined and analyzed.
\end{abstract}

Keywords: Artificial Neural Networks, Unsupervised Learning, Projection Models, Network \& Computer Security, Intrusion Detection, Honeypots.

\section{Introduction}

A network attack or intrusion will inevitably violate one of the three computer security principles — availability, integrity and confidentiality — by exploiting certain vulnerabilities such as Denial of Service (DoS), Modification and Destruction. ${ }^{1}$ One of the most harmful issues of attacks and intrusions, which increases the difficulty of protecting computer systems, is precisely the ever-changing nature of attack technologies and strategies.

For that reason alone, among others, $\operatorname{IDSs}^{2-6}$ have become an essential asset, to complement to the computer security infrastructure of most organizations. In the context of computer networks, an IDS can roughly be defined as a tool designed to detect suspicious patterns that may be related to a network or system attack. Intrusion Detection (ID) is therefore a field that focuses on the identification of attempted or ongoing attacks on a computer system (Host IDS - HIDS) or network (Network IDS - NIDS).

Visual inspection of traffic patterns is an alternative and crucial aspect in network monitoring. ${ }^{7}$ Visualization is a critical issue in the computer network defense environment. It serves to generate a synthetic and intuitive representation of the current situation for the network manager. As a result, several research initiatives have recently applied information visualization to this challenging task. ${ }^{8-11}$ Visualization techniques typically aim to make the 
available statistics supplied by traffic-monitoring systems more understandable in an interactive way. They therefore focus on traffic data as well as on network topology. Regardless of their specific characteristics, these methods all map high-dimensional feature data into a low-dimensional space for visual presentation purposes. The baseline of the novel research presented in this study is that Artificial Neural Networks (ANNs), ${ }^{12-17}$ in general, and unsupervised connectionist models, in particular, can prove quite adequate for the purpose of network data visualization through dimensionality reduction. ${ }^{18-20}$ As a result, unsupervised projection models ${ }^{21,22}$ are applied in the present research for the visualization and subsequent analysis of network traffic data collected by a network of honeypots, also known as a honeynet.

A honeypot has no authorized function or productive value within the corporate network other than to be explored, attacked or compromised. ${ }^{23} \mathrm{~A}$ honeypot should not receive any traffic at all. As a consequence, all the traffic arriving at any honeynet sensor must be considered as suspicious by default. Every packet should be considered as an attack or at least as part of a multi-step attack. Recent works ${ }^{24,25}$ have shown that not every packet is a part of an attack, but most of the traffic corresponds to infected computers, while the rest come from misconfigured devices. Numerous studies propose the use of honeypots to detect automatic large-scale attacks; honeyd ${ }^{26}$ and nepenthes. ${ }^{27}$ among others. The first Internet traffic monitors known as Network Telescopes, Black Holes or Internet Sinks were presented by Moore et $a .^{28}$ This paper advances previous preliminary work ${ }^{29-31}$ on visual analysis of Honeynet data by the same authors, as described in the conclusions.

The remaining five sections of this paper are structured as follows: Sec. 2 briefly describes the topic of computer and network security (mainly Intrusion Detection). Section 3 presents the novel approach proposed for ID while the neural projection and visualization techniques applied in this research are described in Sec. 4. Some experimental results for two different real-life datasets are then presented and comprehensively described in Sec. 5 . Finally, the conclusions of this interdisciplinary study and the future research lines are discussed in Sec. 6.

\section{Computer and Network Security}

This section introduces the main concepts of computer and network security that are the foundations of this novel study.

\subsection{Intrusion detection systems}

Intrusions can be produced by attackers that access the system, by authorized users that attempt to obtain unauthorized privileges, or by authorized users that misuse the privileges given to them. The complexity of such situations increases in the case of distributed network-based systems and insecure networks. Attacks that attempt to access a system through external networks such as the Internet may involve one or several hosts. From a victim's perspective, intrusions are characterized by their manifestations, which might or might not include damage. ${ }^{32}$ Some attacks may produce no manifestations while some apparent manifestations can be produced by system or network malfunctions.

An IDS can be defined as a piece of software that runs on a host, which monitors the activities of users and programs on the same host and/or the traffic on networks to which that host is connected. ${ }^{33}$ The main purpose of an IDS is to alert the system administrator to any suspicious and possibly intrusive event taking place in the system that it is analyzing. Thus, they are designed to monitor and to analyze computer and/or network events, in order to detect suspect patterns that may uncover a system or network intrusion.

Ever since the first studies in this field in the $80 \mathrm{~s}, 3,34$ the accurate, real-time detection of computer and network system intrusions has always been an intriguing problem for system administrators and information security researchers. It may be attributed on the whole to the dynamic nature of systems and networks, the creativity of attackers, the wide range of computer hardware and operating systems and so on. Such complexity arises when dealing with distributed network-based systems and insecure networks such as the Internet. ${ }^{35}$

A standard characterization of IDSs, based on their detection method, or model of intrusions, defines the following paradigms:

- Anomaly-based ID (also known as behaviorbased ID): the IDS detects intrusions by looking 
for activity that differs from the previously defined "normal" behavior of users and/or systems. In keeping with this idea, the observed activity is compared against "predefined" profiles of expected normal usage. It is assumed that all intrusive activities are necessarily anomalous. In real-life environments, instead of their being identical, the set of intrusive activities in some cases intersects the set of anomalous activities. As a consequence, ${ }^{36}$ anomalous activities that are not intrusive are flagged as intrusive (i.e. false positives) and intrusive activities that are not anomalous are not flagged up (i.e. false negatives). Anomaly-based IDSs can support detection of novel (zero-day) attack strategies, but may suffer from a relatively high rate of false positives, ${ }^{37}$

- Misuse-based ID (also known as knowledgebased ID): intrusions are detected by checking activity that corresponds to known intrusion techniques (signatures) or system vulnerabilities. Misuse-based IDSs are therefore commonly known as signature-based IDSs. They detect intrusions by exploiting the available knowledge on specific attacks and vulnerabilities. As opposed to anomaly detection, misuse detection assumes that each intrusive activity can be represented by a unique pattern or signature. ${ }^{38}$ This approach entails one main problem; intrusions with signatures that are not archived by the system can not be detected. As a consequence, a misuse-based IDS will never detect a 0-day attack. ${ }^{38}$ The completeness of such IDSs requires regular updating of their knowledge of attacks.

- Specification-based ID: relying on program behavioral specifications, they reflect system policies that are used as a basis to detect attacks. ${ }^{39}$

\subsubsection{Snort}

Snort, a libpcap-based ${ }^{40}$ lightweight network intrusion detection system, is one of the most widely deployed IDSs. It is a network-based, misuse-based IDS that detects many types of malicious activity in the packet payload that can be characterized in a unique detection signature. Snort functions by collecting packets as quickly as possible and processing them in its detection engine. It is composed of three primary modules: a packet decoder, a detection engine and a logging and alerting subsystem.
Table 1. Snort rules to log all TCP, UDP and ICMP traffic.

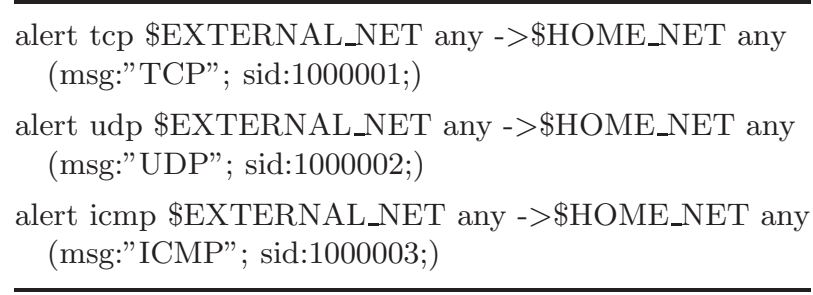

Even though the capabilities of Snort allow indepth analysis of traffic flows, what is of interest in this research is the detection, alerting and logging of the network packets as they are received in the Honeynet system. Snort is used as a network data classifier, without discarding any packet. In that sense, in addition to the default rules of the Snort community, three basic rules that log all TCP, UDP and ICMP traffic are included, as shown in Table 1 .

On the other hand, each incoming packet is inspected and compared with the default rule base. In this way, besides alerting, when the packet matches the three signatures shown above, many of them are also shown to match the Snort rulebase signatures. Therefore, even if a large amount of packets trigger more than one alarm, it facilitates a simple way to separate these alarms into two subsets:

- Alarms triggered by a match with the Snort default rule base. This dataset can be considered as known attack data.

- Alarms that did not match any of the known attack rules, which are considered the unknown data.

These two subsets will allow network administrators to distinguish between known and unknown traffic. This permits the success rate of Snort to be tested, and the unknown traffic to be visualized, to inspect for new and unknown attacks. A clear advantage of using Snort IDS in this study is its ease of use, configuration and the development of new rules.

\subsection{Honeypots and Honeynets}

Recently, two monitoring systems for automatic large-scale attack detection have been proposed: honeypots and network telescopes. Since 1992, 
honeypots have been used to deceive attackers and to learn from the new attacks they accomplish. ${ }^{41-44}$ A honeypot is a decoy system that consists of a vulnerable computing resource that distracts attackers. It is used as an early warning system for new attack proliferations and to ease the later analysis of the attacks (forensics). ${ }^{45}$ When used to monitor activities derived from automatic attacks based on random or pseudo-random scanning, these systems have certain particularities. Unassigned IP addresses are given to these honeypots so that each time a honeypot receives a connection request, it will be categorized as suspicious. Nevertheless, the interaction level of the honeypot is fundamental. The higher the interaction between the honeypot and the attacker (response to TCP connection request for example), the greater the amount of information that is collected, which implies greater levels of knowledge about the attack. A system with a low level of interaction will also be valid to analyze the noise level, detect infected hosts, etc.

One of the most extended classifications of honeypots takes into account their level of interaction. Low interaction honeypots offer limited interaction with attackers and the most common ones only simulate services and operating systems. High interaction honeypots follow a different strategy: instead of using simulated services and operating systems, real systems and applications are used, usually running on virtual machines.

Found somewhere between those two types, medium interaction honeypots also emulate vulnerable services, but leave the operating system to manage the connections with their network protocol stack. Recently, a new type of honeypot has been proposed as a response to the behavioral change observed in the attackers. Instead of waiting for the attackers to reach traditional honeypots, client side honeypots, also known as honeyclients, scan communication channels looking for malware.

The case studies analyzed in this paper focus on medium interaction honeypots.

Different platforms exist as observatories of malicious threats using honeypots. Examples are $\mathrm{NoAH}^{26}$ and SGNET. ${ }^{27}$ This research follows these approaches, based on the application of unsupervised learning to network level packets, collected from a honeypot-based observatory.

\section{A Neural Visualization-Based Approach for Data Monitored by Honeypots}

This study proposes the application of neural projection models for the visualization of network traffic received by honeypots. There are various approaches to the collection of Internet-based attacks, but there is still a lack of techniques that ease comprehension and analysis of the information that is gathered. Visualization techniques have been applied to massive datasets for many years. These techniques are considered a viable approach to information seeking, as humans are able to recognize different features and to detect anomalies by inspecting graphs. ${ }^{46}$ The underlying operational assumption of the proposed approach is largely grounded in the ability to render the high-dimensional traffic data in a consistent yet low-dimensional representation. So, security visualization tools have to map high-dimensional feature data into a low-dimensional space for presentation. One of the main assumptions of this research is that neural projection models will prove satisfactory for the purpose of honeynet data visualization through dimensionality reduction, by analyzing complex high-dimensional datasets received by honeypots.

Projection methods project high-dimensional data points onto a lower dimensional space, in order to identify "interesting" directions in terms of any specific index or projection. Having identified the most interesting projections, the data are then projected onto a lower dimensional subspace plotted into two or three dimensions, which makes it possible to examine the structure with the naked eye. Projection methods can be seen as smart compression tools that map raw, high-dimensional data onto two or three dimensional spaces for subsequent graphical display. By doing so, the structure that is identified through a multivariable dataset may be visually analyzed with greater ease by the naked eye.

Visualization tools can therefore support security tasks in the following way:

- Visualization tools may be understood intuitively (even by inexperienced staff) and require less configuration time than more conventional tools.

- Providing an intuitive visualization of data allows inexperienced security staff to learn more about standard network behavior, which is a key issue in 
ID. ${ }^{47}$ The monitoring task can be then assigned to less experienced security staff.

- As stated in "visualizations that depict patterns in massive amounts of data, and methods for interacting with those visualizations can help analysts prepare for unforeseen events". Hence, such tools can also be used in security training.

- They can work in unison with some other security tools in a complementary way.

As with other machine learning paradigms, an interesting facet of ANN learning is not just that the input patterns may be precisely learned/classified/ identified, but that this learning can be generalized. Whereas learning takes place within a set of training patterns, an important property of the learning process is that the network can generalize its results on a set of test patterns that were not previously learnt. Also, their capability to identify unknown patterns fits the 0-day attack ${ }^{37}$ detection.

Owing to the aforementioned reasons, the present study approaches the analysis of attack data from a visualization standpoint. Thus, some unsupervised neural projection techniques are applied for the visualization of data monitored by honeypots. As previously proposed, ${ }^{20}$ the continuous honeynet dataflow can be split into variable-length segments to reduce depiction time.

\subsection{Previous work}

Even though great effort has been dedicated to ID research, several issues concerning IDS design, development, and performance remain open for further research.

Nevertheless, scant attention has been given to visualization in the ID field, ${ }^{48}$ although visual presentations do assist operators, in general, and security managers, in particular, to interpret large quantities of data. Most IDSs do not provide any way of viewing information other than through lists, aggregates, or trends of raw data. They can generate different alarms when an anomalous situation is detected, broaden monitoring tasks, and increase situational awareness. However, they neither provide a general overview of what is happening in the network, nor support a detailed packet-level inspection ${ }^{9}$ as is the case for honeypots and honeynets.

Some other authors have previously addressed the analysis of traffic data and intrusion detection under the application of $\mathrm{ANN}^{49,50}$ and more particularly projection methods. ${ }^{18,51}$

The underlying idea in this research is not only to detect anomalous situations under data sets monitored by honeypots, but also to visualize protocol interactions and traffic volume. Packet-based ID, that is actually performed in this present research, has several advantages. ${ }^{52}$

Some Exploratory Projection Pursuit (EPP) $)^{53}$ models have been previously applied to the ID field as part of a hybrid intelligent IDS. ${ }^{1{ }^{-20}}$ Unlike previous studies, neural EPP models, are applied here as a complementary tool to IDSs for the first time, to analyze real complex high-dimensional honeynet data sets. Accordingly, the output of both the neural model and Snort are combined, together with some other customized visualizations for comprehensive analysis and understanding of network status.

\section{Neural Visualization Techniques}

The different projection models applied in this study are described in the following subsections.

\subsection{Principal component analysis}

Principal Component Analysis (PCA) is a statistical model (introduced in ${ }^{54}$ and independently in ${ }^{55}$ ), which describes the variation in a set of multivariate data in terms of a set of uncorrelated variables, each of which is a linear combination of the original variables.

Its goal is to derive new variables, in decreasing order of importance, that are linear combinations of the original variables and are uncorrelated with each other.

\subsection{Cooperative maximum likelihood Hebbian learning}

Exploratory Projection Pursuit (EPP) ${ }^{53}$ is a more recent statistical method aimed at solving the difficult problem of identifying structure in high dimensional data. It does this by projecting the data onto a low dimensional subspace in which we search for the data structure by eye. However, not all projections will reveal this structure equally well. It therefore defines an index that measures how "interesting" a given projection is, and then represents the data in terms of projections that maximize the index. 
The first step for EPP is to define which indexes represent interesting directions. "Interestingness" is usually defined with respect to the fact that most projections of high-dimensional data give almost Gaussian distributions. ${ }^{56}$ Thus, in order to identify "interesting" features in data, it is appropriate to look for those directions onto which the dataprojections are as far from the Gaussian as possible.

Two simple measures of deviation from a Gaussian distribution are based on the higher order moments of the distribution. Skewness is based on the normalized third moment and measures the deviation of the distribution from bilateral symmetry. Kurtosis is based on the normalized fourth moment and measures the heaviness of the tails of a distribution. A bimodal distribution will often have a negative kurtosis, which will therefore signal that a particular distribution shows evidence of clustering.

If a Gaussian distribution with mean $a$ and variance $x$ is as interesting as a Gaussian distribution with mean $b$ and variance $y$, then such information may be removed from the data (sphering). In effect, the second order structure can obscure structures of a higher order that are more interesting.

Cooperative Maximum Likelihood Hebbian Learning (CMLHL) $)^{21,57}$ is based on Maximum Likelihood Hebbian Learning (MLHL), ${ }^{21,58}$ an EPP connectionist model. CMLHL includes lateral connections ${ }^{57,59}$ derived from the Rectified Gaussian Distribution (RGD). ${ }^{60}$ The RGD is a modification of the standard Gaussian distribution in which the variables are constrained to be non-negative, enabling the use of non-convex energy functions. The CMLHL architecture is depicted in Fig. 1, where lateral connections are highlighted.

The lateral connections used by CMLHL are based on the cooperative distribution mode that is closely spaced along a non-linear continuous manifold. In consequence, the resultant net can find the independent factors of a dataset in a way that captures some type of global ordering.

Considering an N-dimensional input vector $(x)$, an M-dimensional output vector $(y)$ and with $W_{i j}$ being the weight (linking input $j$ to output $i$ ), CMLHL can be expressed as:

Feed-forward step:

$$
y_{i}=\sum_{j=1}^{\mathbf{N}} W_{i j} x_{j}, \quad \forall i
$$

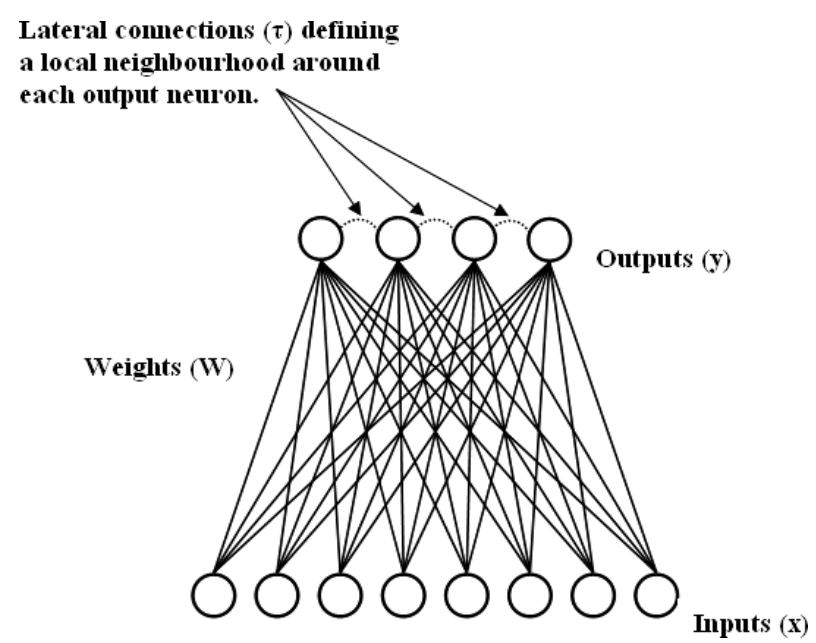

Fig. 1. CMLHL: lateral connections between neighboring output neurons.

Lateral activation passing:

$$
y_{i}(t+1)=\left[y_{i}(t)+\tau(b-A y)\right]^{+}
$$

Feedback step:

$$
e_{j}=x_{j}-\sum_{i=1}^{M} W_{i j} y_{i}, \quad \forall j
$$

Weight change:

$$
\Delta W_{i j}=\eta \cdot y_{i} \cdot \operatorname{sign}\left(e_{j}\right)\left|e_{j}\right|^{p}
$$

where $\eta$ is the learning rate, $\tau$ is the "strength" of the lateral connections, $b$ the bias parameter and $p$ is a parameter related to the energy function. ${ }^{21,57,58}$

$A$ is a symmetric matrix used to modify the response to the data, the effect of which is based on the relation between the distances between the output neurons. It is based on Cooperative Distribution, but to speed learning up, it can be simplified to:

$$
A(i, j)=\delta_{i j}-\cos (2 \pi(i-j) / M)
$$

where, $\delta_{i j}$ is the Kronecker delta.

It has already been demonstrated that CMLHL can itself successfully perform data visualization. It was initially applied in the field of artificial vision $^{57,59}$ and then to some other problems. ${ }^{61-63}$

\subsection{Curvilinear Component Analysis}

Curvilinear Component Analysis (CCA $)^{56}$ is a nonlinear dimensionality reduction method. Developed 
as an improvement on the SOM, it tries to circumvent the limitations inherent in previous linear models such as PCA.

The principle of CCA is a self-organized neural network performing two tasks: a vector quantization of the submanifold in the data set (input space) and a nonlinear projection of these quantizing vectors toward an output space, providing a revealing view of the way in which the submanifold unfolds. Quantization and nonlinear mapping are separately performed by two layers of connections: firstly, the input vectors are forced to become prototypes of the distribution using a vector quantization (VQ) method; then, the output layer builds a nonlinear mapping of the input vectors according to Euclidean distances.

In the vector quantization step, the input vectors $\left(x_{i}\right)$ are forced to become prototypes of the distribution by using competitive learning and the regularization method ${ }^{57}$ of vector quantization. Thus, this step, which is intended to reveal the submanifold of the distribution, regularly quantizes the space covered by the data, regardless of the density. Euclidean distances between these input vectors $\left(X_{i j}=d\left(x_{i}, x_{j}\right)\right)$ are applied, as the output layer has to build a nonlinear mapping of the input vectors. The corresponding distances in the output space are also used $\left(Y_{i j}=d\left(y_{i}, y_{j}\right)\right)$.

Perfect matching is not possible at all scales when the manifold is "unfolding", so a weighting function $\left(F\left(Y_{i j}, \lambda_{y}\right)\right)$ is introduced, yielding the quadratic cost function:

$$
E=\frac{1}{2} \sum_{i} \sum_{j \neq i}\left(X_{i j}-Y_{i j}\right)^{2} F\left(Y_{i j}, \lambda_{y}\right)
$$

where $\lambda_{y}$ is a user-tuned parameter allowing an interactive selection of the scale at which the unfolding takes place.

As regards its goal, the projection part of CCA is similar to other nonlinear mapping methods, in that it minimizes a cost function based on interpoint distances in both input and output spaces. Instead of moving one of the output vectors $\left(y_{i}\right)$ according to the sum of the influences of every other $y_{j}$ (as would be the case for a stochastic gradient descent), CCA proposes pinning down one of the output vectors $\left(y_{i}\right)$ "temporarily", and moving all the other $y_{j}$ around, disregarding any interactions between them. Accordingly, the proposed "learning" rule can be expressed as:

$$
\Delta y_{j}=\alpha(t) F\left(Y_{i j}, \lambda_{y}\right)\left(X_{i j}-Y_{i j}\right) \frac{y_{j}-y_{i}}{Y_{i j}} \quad \forall j \neq i
$$

where $\lambda$ is the step size that decreases over time.

\subsection{Self-organizing map}

The Self-Organizing Map (SOM) ${ }^{65,66}$ was developed as a visualization tool for representing high dimensional data on a low dimensional display. It is also based on the use of unsupervised learning. However, it is a topology preserving mapping model rather than a projection architecture.

Typically, the array of nodes is one or twodimensional, with all nodes connected to the $N$ inputs by an $N$-dimensional weight vector. The selforganization process is commonly implemented as an iterative on-line algorithm, although a batch version also exists. An input vector is presented to the network and a winning node, the weight vector of which, $W_{c}$, the closest (in terms of Euclidean distance) to the input, is chosen:

$$
c=\arg \min _{i}\left(\left\|\mathbf{x}-W_{i}\right\|\right)
$$

Data vectors are quantized to the reference vector in the map that is closest to the input vector. The weights of the winning node and the nodes close to it are then updated to move closer to the input vector. There is also a learning rate parameter $(\eta)$ that usually decreases as the training process progresses. The weight update rule for $N$ inputs is defined as follows:

$$
\Delta W_{i}=\eta h_{c i}\left[\mathbf{x}-W_{i}\right], \quad \forall i \in N^{(c)}
$$

where $W_{i}$ is the weight vector associated with neuron $i, \mathbf{x}$ is the input vector, and $h$ is the neighborhood function.

When this algorithm is sufficiently well iterated, the map self-organizes to produce a topologypreserving mapping of the lattice of weight vectors to the input space based on the statistics of the training data.

\section{Experimental Study}

Researchers usually make use of well-known attack datasets such as the DARPA dataset ${ }^{6-69}$ or the KDD Cup '99 sub-dataset ${ }^{70,71}$ in order to validate the systems they have developed. However, these 
data are simulated, non-validated and irregular, so they are not fully reliable. ${ }^{72,73}$ Even if the results obtained by such systems are good, no one can ensure that their algorithms will make the system more secure or will detect real attacks. This is the main reason for using two real traffic data sets coming from a running honeynet in this research.

The experimental work has been conducted by using real data traffic received by the Euskalert network. ${ }^{74}$ These data are depicted through different neural projection and visualization techniques in order to discover real attack behavior and strategies.

The Euskalert project ${ }^{74}$ has deployed a network of honeypots in the Basque Country (northern Spain), where eight companies and institutions have installed one of the project's sensors behind the firewalls of their corporate networks. The honeypot sensor transmits all the traffic received to a database via a secure communication channel. These partners can consult information relative to their sensor as well as general statistics on the project's website. Once a large amount of data has been collected, the available information can be used to analyze attacks received by the honeynet at network and application level.

Euskalert is a distributed honeypot network based on a Honeynet GenIII architecture. ${ }^{53}$ The
Eusakalert architecture is shown in Fig. 2. The various sensors installed in corporate networks of the different participants are shown on the left in Fig. 2.

Each sensor has a permanently established encrypted connection (using different virtual private networks) to a tunnel server, which is in the DMZ (Demilitarized Zone) of Mondragon University. Any attack on one of the sensors is redirected through these tunnels to reach the Honeypot (right side of Fig. 2), which is responsible for responding to any connection attempt. The traffic also passes through a server responsible for collecting all the information, which is then displayed on the Web platform. ${ }^{74}$

This honeypot system receives about 164 packets a day on average. All the incoming traffic is analyzed by the Snort IDS, and an alert is launched whenever a packet matches a known signature.

The following features were extracted from each one of the records in the dataset:

- Time: The time when the attack was detected. Difference in relation to the first attack in the dataset (in minutes).

- Protocol: Either TCP, UDP or ICMP (codified as three binary features).

- Ip_len: Number of bytes in the packet.

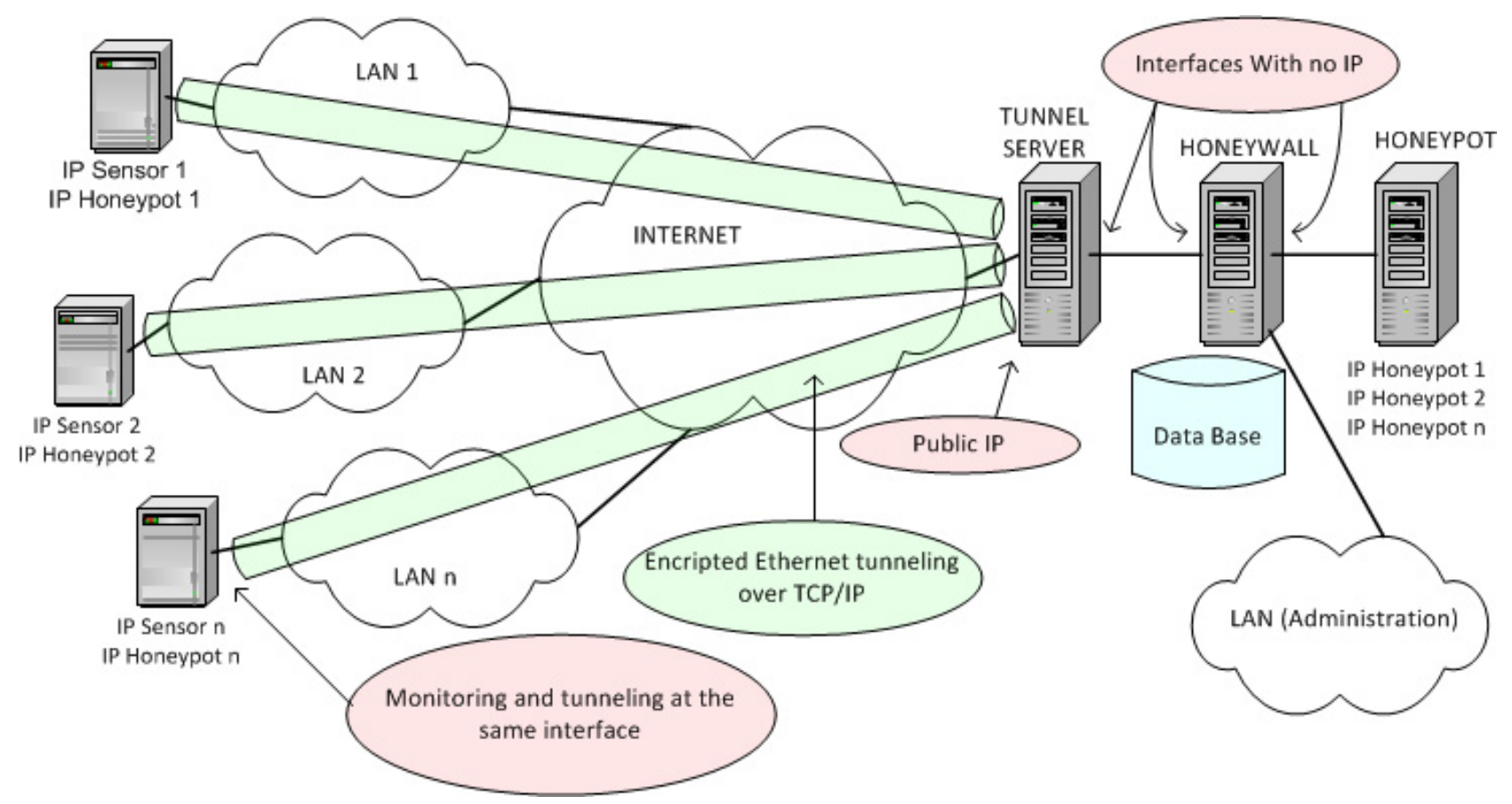

Fig. 2. Architecture of the Euskalert network. 
- Source Port: Number of the port from which the source host sent the packet. In the case of the ICMP protocol, this represents the ICMP type field.

- Destination Port: Destination host port number to which the packet was sent. In the case of the ICMP protocol, this represents the ICMP type field.

- Flags: Control bits of a TCP packet, which contains 8 1-bit values.

Two different real-life case studies were analyzed in this research as attack behavior may change over time. First, a one-month snapshot of data received (February, 2010) was analyzed, to observe its internal structure. A five-month period (February-June, 2010) was subsequently analyzed to see how attacks had changed and to discover new trends.

The projection models introduced the earlier sections have been applied to these two case studies, the results of which are shown and described in the following subsections.

\subsection{Case study 1: A 1-month dataset}

For this real case study, the logs coming from Euskalert and Snort were collected over one month (February, 2010). Figure 3 shows the traffic volume in terms of the number of packets received for that period of time. The amount of daily network traffic received changes from day to day. Most of the traffic is malicious, thus it does not follow any predefined pattern or distribution, as the traffic volume is unpredictable. In this case, days 11/02/2010 and 14/02/2010 might reflect a new worm outbreak, or a Denial of Service attack. Furthermore, occasional breaks occur in the connection between the server and the sensors that collect the traffic, which

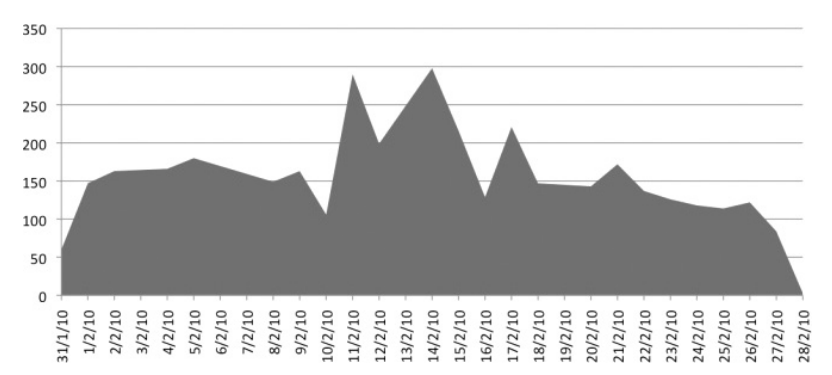

Fig. 3. Temporal distribution of the traffic volume in terms of number of packets captured by Euskalert during February 2010.
Table 2. Characterization of data traffic captured by Euskalert, during February, 2010.

\begin{tabular}{|c|c|c|}
\hline Signature & \# Packets & $\%$ of traffic \\
\hline Unknown Traffic & 3404 & 89.62 \\
\hline $\begin{array}{l}\text { POLICY Reserved IP Space } \\
\text { Traffic - Bogon Nets } 2\end{array}$ & 127 & 3.34 \\
\hline $\begin{array}{l}\text { WORM Allaple ICMP } \\
\text { Sweep Ping Inbound }\end{array}$ & 58 & 1.52 \\
\hline ICMP PING & 75 & 1.97 \\
\hline $\begin{array}{l}\text { Wormledge, microsoft-ds, } \\
\text { smb directory packet } \\
\text { (port 445) }\end{array}$ & 34 & 0.89 \\
\hline $\begin{array}{l}\text { Wormledge, KRPC } \\
\text { Protocol, BitTorrent }\end{array}$ & 11 & 0.28 \\
\hline $\begin{array}{l}\text { Wormledge, NetBios Session } \\
\text { Service (port 139) }\end{array}$ & 7 & 0.18 \\
\hline $\begin{array}{l}\text { Wormledge, NetBios Name } \\
\text { Query (udp port 137) }\end{array}$ & 7 & 0.18 \\
\hline $\begin{array}{l}\text { Wormledge, Microsoft RPC } \\
\text { Service, dce endpoint } \\
\text { resolution (port 135) }\end{array}$ & 7 & 0.18 \\
\hline $\begin{array}{l}\text { WEB-IIS view source via } \\
\text { translate header }\end{array}$ & 6 & 0.15 \\
\hline $\begin{array}{l}\text { SCAN LibSSH Based SSH } \\
\text { Connection }\end{array}$ & 5 & 0.13 \\
\hline
\end{tabular}

also causes a drop in traffic (see 10/02/2010 or 28/02/2010).

The February 2010 dataset contains a total of 3798 packets, including TCP, UDP and ICMP traffic received by the distributed honeypot sensors. The characterization of the traffic in the dataset is shown in Table 2. The table shows which alerts have been triggered in that period of time and their percentage. Those signatures starting with "Wormledge" are automatically generated and are not present in the default signature database.

From this dataset, it may be said that a misuse detection-based IDS such as Snort is only capable of identifying about $10.38 \%$ of bad-intentioned traffic. Furthermore, it was demonstrated that only $2 \%$ of the unsolicited traffic was identified by the IDS when automatically generated signatures were included from a previous work. ${ }^{24}$ Thus, a more exhaustive data analysis is needed in order to discover the internal structure of the remaining $90 \%$ of the traffic. Explaining the behavior of the unknown traffic is a difficult task that must be performed to better protect computer networks and systems. Several neural projection models were applied to acquire more 


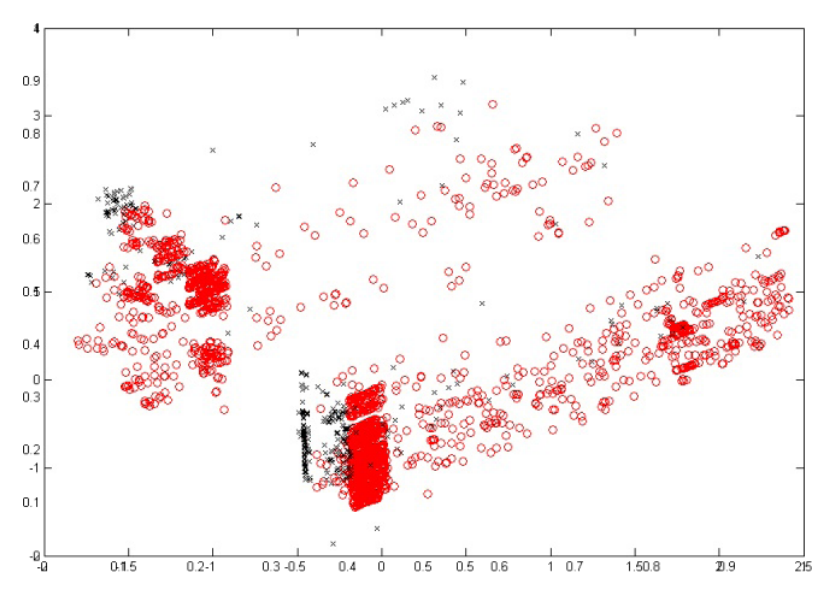

Fig. 4. CMLHL projection of 1-month data - Snort output. Parameters: number of iterations $=10,000$, learning rate $=0.0208, p$ parameter $=2.1429$, and $\tau$ parameter $=0.067$.

knowledge, and the results and conclusions are shown in the following sub-sections.

The data in the visualizations are depicted by different colors and shapes, according to their original features. In the projections that are shown (Figs. 4 to 7 ), the axes are combinations of the features contained in the original datasets. Then, the $\mathrm{X}$ and $\mathrm{Y}$ axes of the projections can not be associated with a unique original feature. ${ }^{21,57}$

\subsubsection{CMLHL projections}

The CMLHL-training parameter values for the projections in this section were: number of

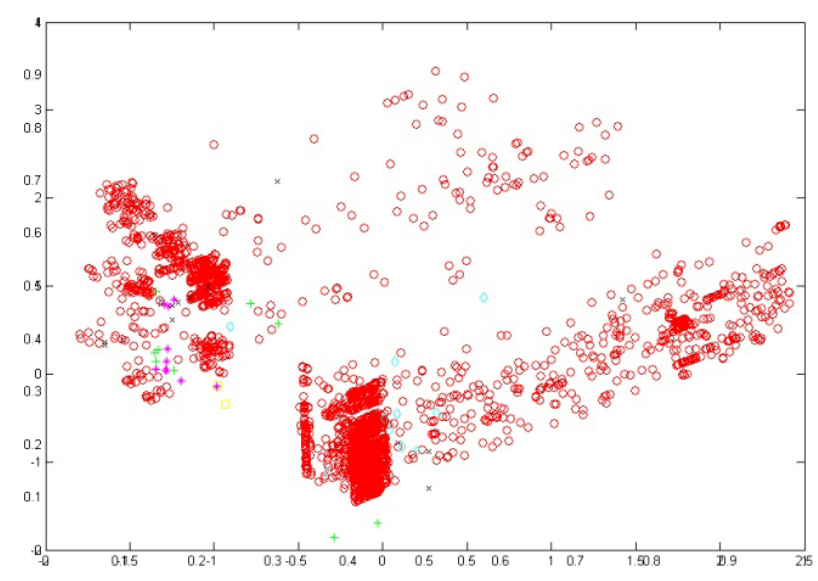

Fig. 5. CMLHL projection of 1-month data - IP length. Parameters: number of iterations $=10,000$, learning rate $=0.0208, p$ parameter $=2.1429$, and $\tau$ parameter $=0.067$.

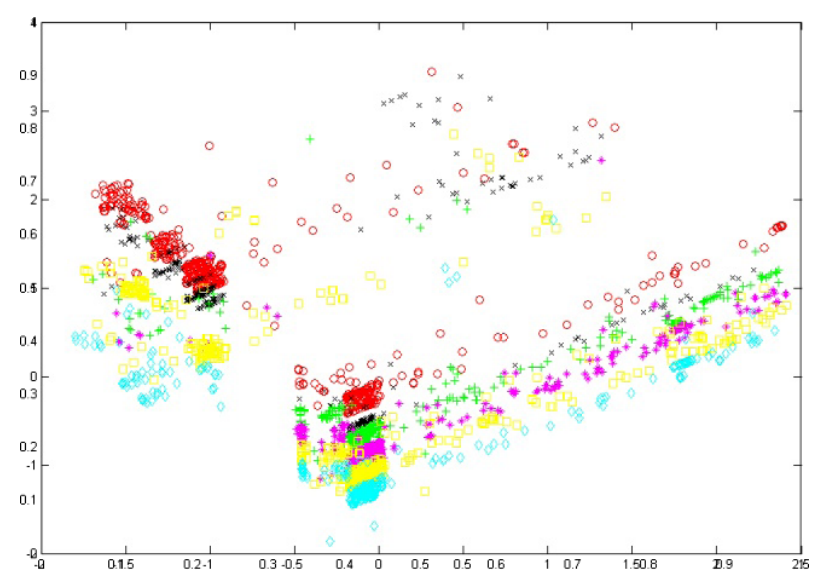

Fig. 6. CMLHL projection of 1-month data Timestamp. Parameters: number of iterations $=10,000$, learning rate $=0.0208, p$ parameter $=2.1429$, and $\tau$ parameter $=0.067$.

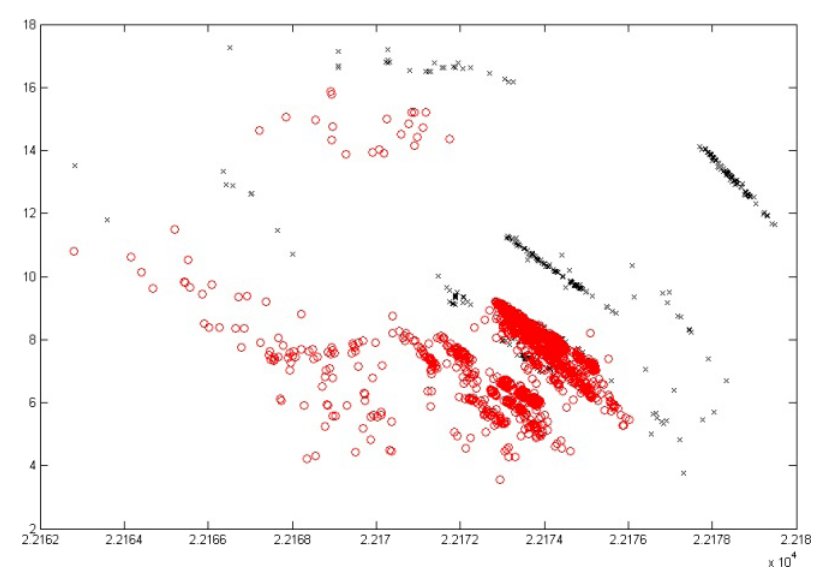

Fig. 7. CCA projection of 1-month data - Snort output. Parameters: standardized Euclidian distance, lambda $=230,000$, alpha $=0.5$ and 10 epochs.

iterations $=10,000$, learning rate $=0.0208, p$ parameter $=2.1429$, and $\tau$ parameter $=0.067$.

Figure 4 shows the CMLHL projection of the output generated by Snort. Packets that triggered alarms are depicted as black crosses, while packets identified as unknown are depicted as red circles.

An analysis of this projection (Fig. 4) confirms the poor detection performance of Snort IDS when filtering honeypot traffic. CMLHL provides a way of differentiating known from unknown traffic for the naked eye. Most of the traffic corresponds to unknown packets, or at least to traffic that Snort is not capable of identifying using all of its predefined rule sets. 
The CMLHL projection, in Fig. 5, depicts the packets in terms of the detection timestamp (in minutes): red circles from 0 to 6692 ; black crosses from 6693 to 13384; green pluses from 13385 to 20076; magenta stars from 20077 to 26768 ; yellow squares from 26769 to 33460 ; and cyan diamonds from 33461 to 40148 .

The temporal evolution of the traffic in that month shows that similar traffic patterns repeat over time, as almost every cluster has a similar shape. This occurs for both known and unknown traffic (shown in Fig. 4). It can be concluded that anomalous or unknown behavior is not a one-off event, but a recurring pattern in time.

The CMLHL projection, in Fig. 6, depicts the packets in terms of their IP length (in bits): red circles from 28 to 273 ; black crosses from 274 to 519 ; green pluses from 520 to 765 ; magenta stars from 766 to 1011; yellow squares from 1012 to 1257 ; and cyan diamonds from 1258 to 1500 .

Most of the traffic is composed of small packets, but it can also be observed that very large packets are received by the honeypot sensors. Attackers must create specially prepared packets, in order to overflow the listening service's memory buffers and stacks first, and then execute arbitrary commands later. This payload, known as shellcode, can have a large size. These are synonyms for reception of malware, exploitation of vulnerabilities, and DoS attacks.

All those visualizations are in general very helpful information for explaining the different traffic behavior on their systems and assist security administrators to interpret the data.

\subsubsection{Comparative study}

The CMLHL projections are compared with two other dimensionality-reduction models (CCA and SOM). Several experiments were required to tune CCA to different options and parameters: initialization, epochs and distance criterion, among others. In the case of SOM, other parameters, such as grid size, batch/online training, initialization, number of iterations and distance criterion were tuned. Only the best results (from the standpoint of the projection) for each model, which were obtained after the tuning stage, are included in this section.

\subsubsection{Curvilinear component analysis}

Figure 7 shows the CCA projection of case study 1 of the Snort output. The following parameters were tuned: alpha, lambda, number of epochs and distance criterion. The final selected parameter values were: standardized Euclidian distance, lambda $=230,000$, alpha $=0.5$ and 10 epochs.

This projection (Fig. 7) shows a visual explanation of the distribution of packets identified by Snort and those which are not. CCA is much more resource demanding than the other models as the pair-wise distance matrix must be calculated.

\subsubsection{Self-organizing map}

Finally, the SOM was also applied to the 1-month dataset. Figure 8 shows the SOM map of Case Study 1 by depicting the Snort output. The two different Snort outputs $(1=$ triggered alarms, $0=$ no triggered alarms) are assigned to SOM neurons, in order to analyze the resulting maps.

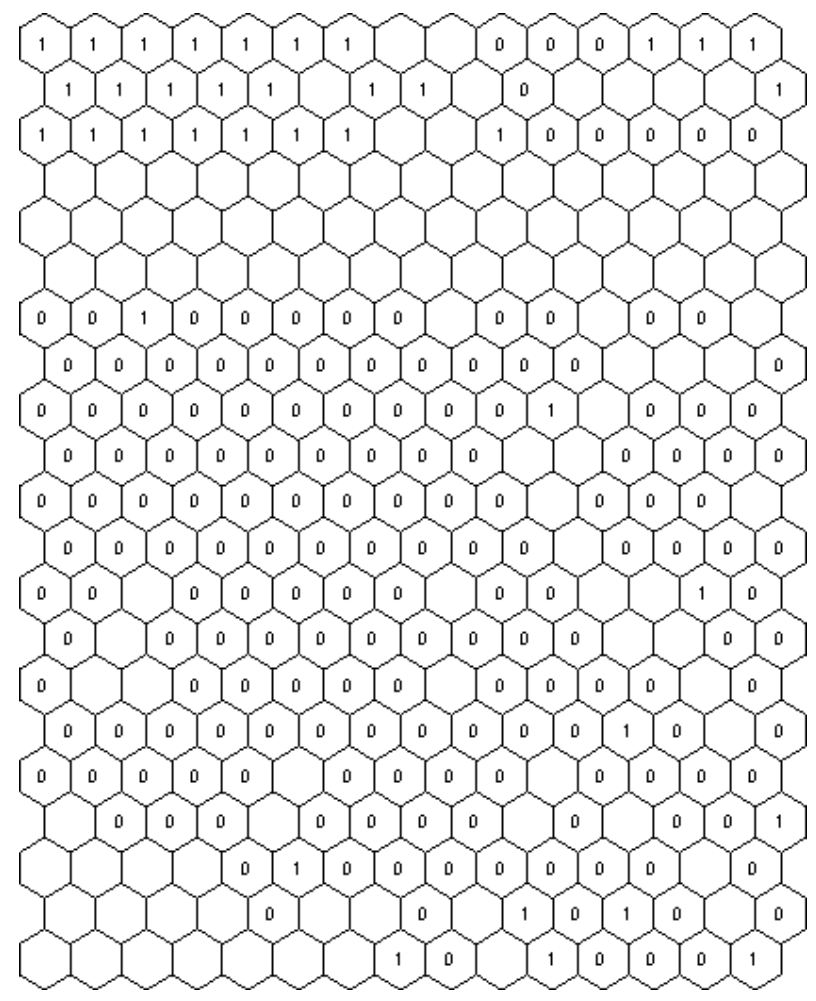

Fig. 8. SOM mapping of 1-month data - Snort output. Parameters: linear initialization, batch training, hexagonal lattice, "Cut Gaussian" neighborhood function, and grid size (determined by means of a heuristic formula) $=15 \times 21$. 
In the case of the SOM, the following options and parameters, among others, were tuned: grid size, batch/online training, initialization, number of iterations and distance criterion among others. The parameter values were: linear initialization, batch training, hexagonal lattice, "Cut Gaussian" neighborhood function, and grid size (determined by means of a heuristic formula $)=15 \times 21$.

After analyzing this mapping, it can be concluded that SOM is not able to cluster the data distinguishing the traffic classification of Snort (alarm/no alarm). The cluster in the upper left section (Fig. 8) is the only one that identifies traffic of only one class (packets that triggered an alarm), while the other ones identify traffic from the two classes.

\subsection{Case study 2: A 5-month real dataset}

For this experiment, we have analyzed the logs coming from Euskalert and Snort collected over five months starting from February, 2010. Figure 9 shows the traffic volume in terms of number of packets received for that period of time.

The dataset contains a total of 22,601 packets, including TCP, UDP and ICMP traffic received by the distributed honeypot sensors. The characterization of the traffic in this dataset, in Table 3, shows which alerts were triggered in that period of time and their percentage. Signatures starting with "Wormledge" were automatically generated and are not present in the default Snort signature database. As Table 3 shows, the largest group of signatures were generated for unknown packets (both TCP, UDP and ICMP), and the automatically generated signatures from a previous work. ${ }^{24}$

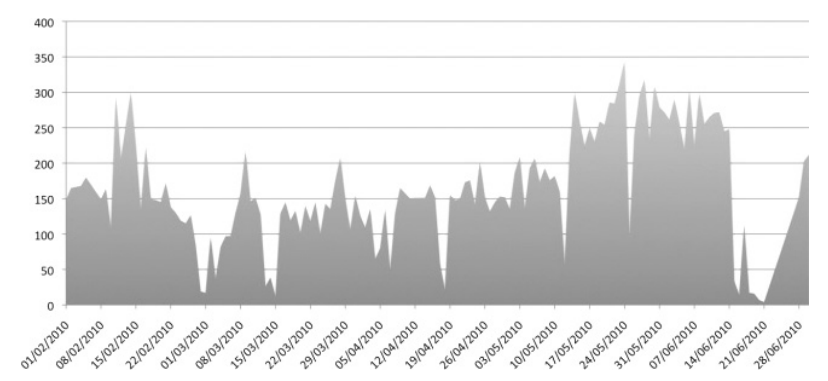

Fig. 9. Temporal distribution of the traffic volume in terms of number of packets captured by Euskalert from February to June, 2010.
Table 3. Characterization of traffic data captured by Euskalert, from February to June, 2010.

\begin{tabular}{|c|c|c|}
\hline Signature & \# Packets & $\%$ of traffic \\
\hline Unknown TCP packet & 19096 & 84.49183664 \\
\hline $\begin{array}{l}\text { Reserved IP Space } \\
\text { Traffic - Bogon Nets }\end{array}$ & 1071 & 4.738728375 \\
\hline Unknown packet & 741 & 3.27861599 \\
\hline Unknown UDP packet & 397 & 1.756559444 \\
\hline ICMP ping & 290 & 1.283129065 \\
\hline $\begin{array}{l}\text { WORM Allaple ICMP } \\
\text { Sweep Ping Inbound }\end{array}$ & 251 & 1.110570329 \\
\hline $\begin{array}{l}\text { Wormledge, KRPC } \\
\text { Protocol, BitTorrent }\end{array}$ & 99 & 0.438033715 \\
\hline $\begin{array}{l}\text { Wormledge, Slammer } \\
\text { Worm }\end{array}$ & 62 & 0.274324145 \\
\hline $\begin{array}{l}\text { Wormledge, Microsoft-ds, } \\
\text { smb directory packet } \\
\text { (port 445) }\end{array}$ & 62 & 0.274324145 \\
\hline $\begin{array}{l}\text { Wormledge, } \\
\text { MS-SQL-Service(port } \\
\text { tcp 1433) }\end{array}$ & 58 & 0.256625813 \\
\hline ICMP PING speedera & 40 & 0.176983319 \\
\hline $\begin{array}{l}\text { Wormledge, NetBios } \\
\text { Name Query (udp port } \\
137 \text { ) }\end{array}$ & 35 & 0.154860404 \\
\hline $\begin{array}{l}\text { Wormledge, Possible SQL } \\
\text { Snake/Spida Worm }\end{array}$ & 34 & 0.150435821 \\
\hline $\begin{array}{l}\text { Wormledge, NetBios } \\
\text { Session Service (port } \\
\text { 139) }\end{array}$ & 34 & 0.150435821 \\
\hline $\begin{array}{l}\text { SIP TCP/IP message } \\
\text { flooding directed to SIP } \\
\text { proxy }\end{array}$ & 33 & 0.146011238 \\
\hline
\end{tabular}

From this dataset, it may be said that a misuse detection-based IDS such as Snort is only capable of identifying less than $3.75 \%$ (847 packets out of 22,601 ) of bad-intentioned traffic. Compared to the initial month, the percentage of identified packets is smaller in this case study. There are two reasons that might explain this fact. Firstly, there is an element of randomness, as neither can the volume that will generate old and new malware be known, nor traffic from misconfigured devices, nor the nature of that traffic (known/unknown classification by Snort IDS). Traffic bursts of any type may occur at any time. Secondly, it is related with the time that elapses until the Snort signatures are updated. The older the signatures are, the greater the likelihood of unknown traffic. Another observation in this dataset is the higher average volume traffic found in this period. 
This occurred because of traffic congestion, but especially due to the fact that a new sensor was added to the Euskalert platform. Thus, a more in-depth analysis of the data is needed, in order to discover the internal structure of the remaining $96.25 \%$ of the traffic data set. As in case study 1, neural unsupervised models were applied, in order to explain the behavior of the unknown traffic.

\subsubsection{CMLHL projections}

CMLHL was applied, in order to analyze the dataset described above and to identify its inner structure. The CMLHL-training parameter values for the projections in this section were: number of iterations $=$ 30,000 , learning rate $=0.01, p$ parameter $=1.22$, and $\tau$ parameter $=0.13137$.

Figure 10 shows the CMLHL projection by considering the output generated by Snort. Packets that triggered an alarm are depicted as black crosses, while packets that were not identified as anomalous are depicted as red circles.

Visualization of packets using Snort output shows the detection rate of the most widely used misusebased IDS. The only ones detected by Snort have black crosses, where all of the records constitute a suspicious activity by default. It is therefore important to use additional supporting systems, such as the visualization aids proposed in this study, in order to give a more comprehensive picture of what is actually happening and how an IDS is performing.

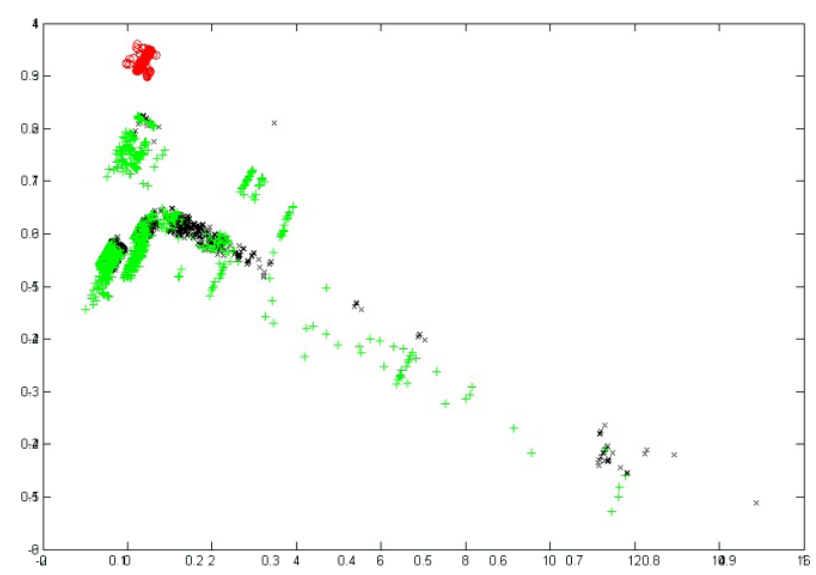

Fig. 10. CMLHL projection of 5-month data - Snort output. Parameters: number of iterations $=30,000$, learning rate $=0.01, p$ parameter $=1.22$, and $\tau$ parameter $=0.13137$.

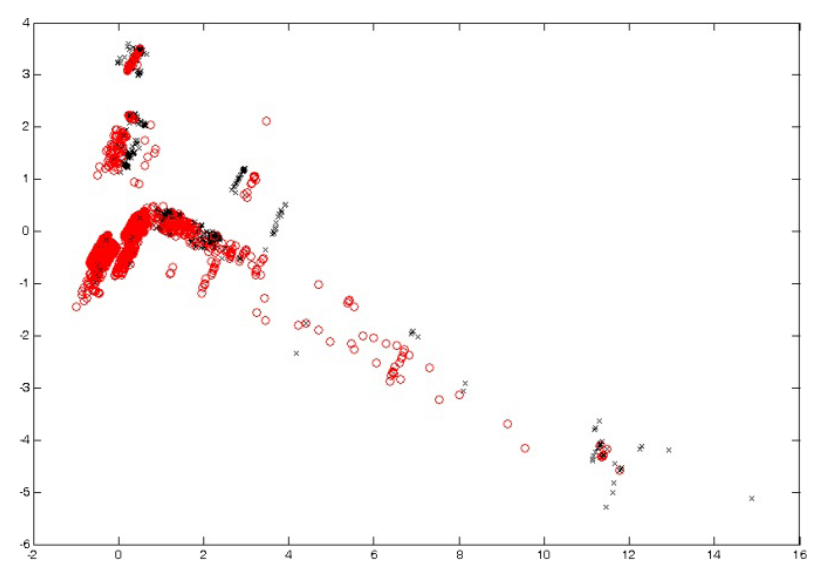

Fig. 11. CMLHL projection of 5-month data - Destination Port. Parameters: number of iterations $=30,000$, learning rate $=0.01, p$ parameter $=1.22$, and $\tau$ parameter $=0.13137$.

The CMLHL projection, in Fig. 11, depicts the packets in terms of the detection timestamp; red circles from 0 to 27044 minutes; black crosses from 27045 to 54089; green pluses from 54090 to 81134; magenta stars from 81135 to 108179 ; yellow squares from 108180 to 135224 ; and cyan diamonds from 135225 to 162267 .

It can be observed that groups are highly overlapped, which means that the temporal distribution of attacks is very homogenous, so they constantly repeat over time. If we look at some of the attacks carefully, we still see very old worm instances such as Slammer or Blaster (see Table 3).

Figure 12 shows the CMLHL projection by considering different ranges of the destination port to depict the packets; red circles correspond to ICMP type codes at 3 and at 8; black crosses correspond to known application listening services from 0 to 1023 (excluding 3 and 8); and green pluses correspond to non-privileged ports from 1024 to 54612 . Had we chosen particular port numbers of commonly exploited services, we would have depicted too many clusters. Choosing the privileged, non-privileged and ICMP bands provides a clear view of the nature of the traffic that is observed.

This visualization shows that Euskalert receives packets and connection attempts to ports above 1023. We find two possible explanations of this observation. On the one hand, there are attack attempts to applications listening on ports above 1023. In this case we should focus on these ports and create 


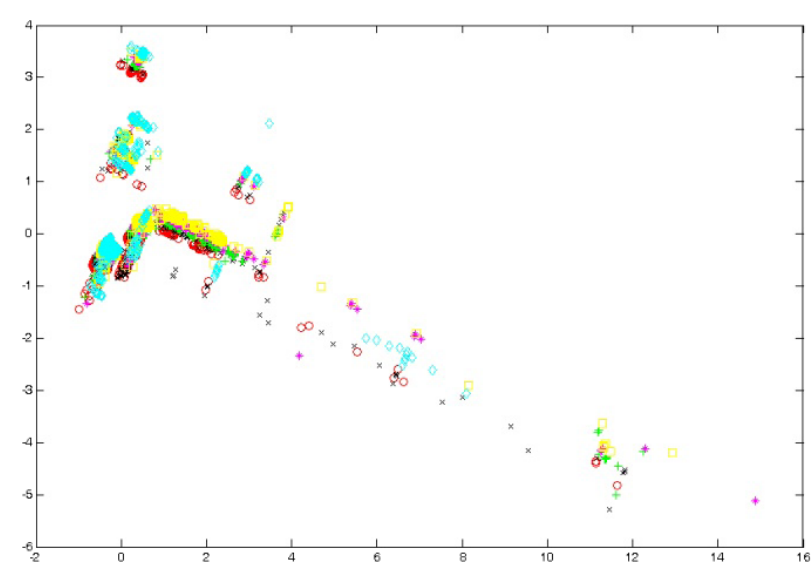

Fig. 12. CMLHL projection of 5-month data Timestamp. Parameters: number of iterations $=30,000$, learning rate $=0.01, p$ parameter $=1.22$, and $\tau$ parameter $=0.13137$.

a new simulated service for that application, if we find any prevalence. On the other hand, backscatter is received, as this port is the source port of the attacker.

\subsubsection{Comparative study}

As in case study 1, the CMLHL projections are compared with those of other dimensionality-reduction models; PCA and MLHL in this case.

\subsubsection{Principal component analysis}

The PCA projection of Case Study 2, in Fig. 13, depicts the packets in terms of the time feature: red circles from 0 to 27044 minutes; black crosses from

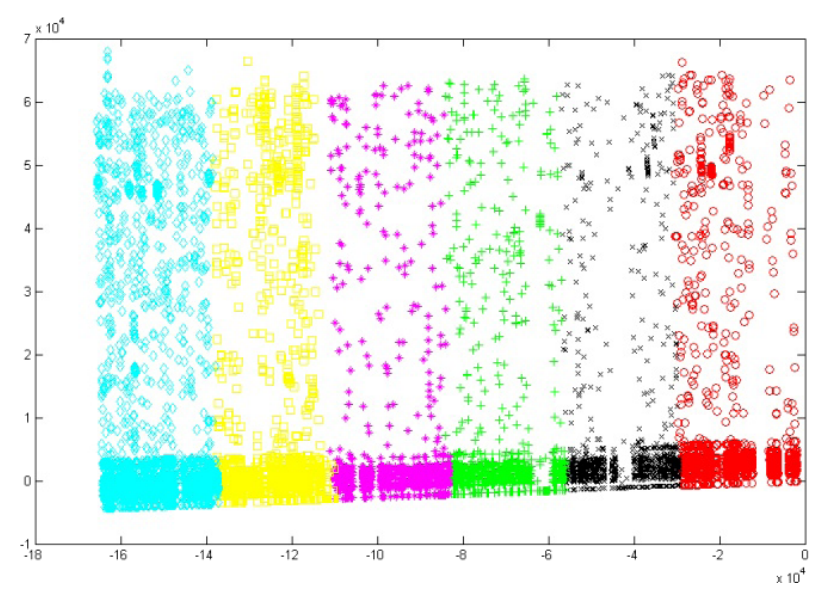

Fig. 13. PCA projection of 5-month data - Time.

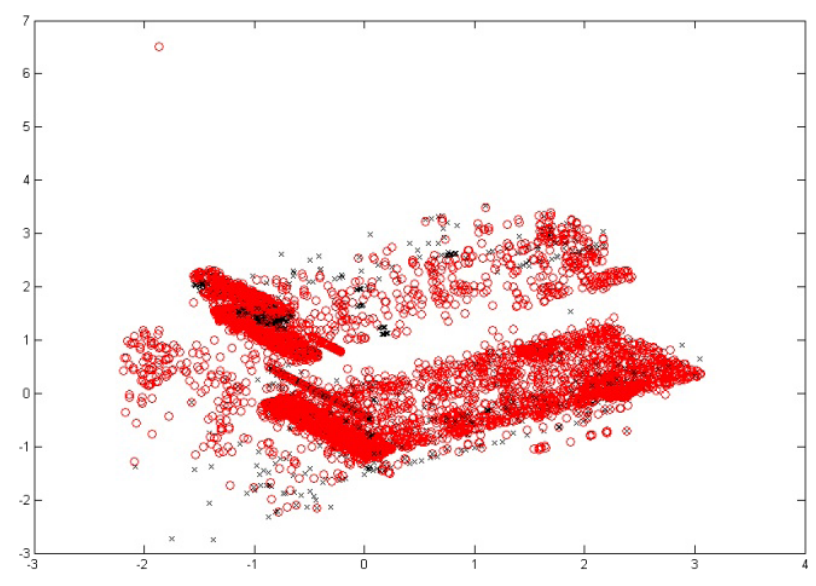

Fig. 14. MLHL projection of 5-month data - Snort output. Parameters: number of iterations $=35,000$, learning rate $=0.02$, and $p$ parameter $=0.9$.

27045 to 54089; green pluses from 54090 to 81134; magenta stars from 81135 to 108179 ; yellow squares from 108180 to 135224; and cyan diamonds from 135225 to 162267 . The two first principal components amount to $96.87 \%$ of original data variance.

Analysis of this visualization allows us to affirm that it offers a clear representation of the conclusions that we have observed, as the packet distribution in time is similar.

\subsubsection{Maximum likelihood Hebbian learning}

The MLHL-training parameter values for the projection in this section were: number of iterations $=35,000$, learning rate $=0.02$, and $p$ parameter $=0.9$.

Figure 14 shows the MLHL projection of the Snort output (Case Study 2). This visualization shows the conclusions obtained from Fig. 10 in sharp relief. It demonstrates that most of the traffic reaching the honeypot remains unexplained, although in the MLHL projection there are no clearly defined clusters.

\section{Conclusions and Future Work}

Apart from the previously stated conclusions, regarding each dataset analysis, some other (more general) conclusions are provided in this section. These conclusions are different from the ones in previous studies, mainly because one of the dataset analysed is larger than any of the preceding ones. In consequence, new attacks and situations are 
considered. Moreover, a comprehensive comparative study is provided, compressing results from a wide range of projection/visualization models.

After comparing the different projections obtained in this study, it can be concluded that CMLHL provides a sparser and clearer representation than the other projection methods. This facilitates intuitive visualization of the Honeynet data, in which the general structure of these data can be seen and interpreted. Advancing previous work, the visualizations obtained through CMLHL incorporating Snort output, provide insight into the captured honeynet data, and useful knowledge of potential network attacks. The application of this neural model generates a depiction of the captured traffic, rather than network statistics or topology, as some previous works have done.

A further contribution of this study is the fact that CMLHL is capable of analyzing large volumes of data, keeping the visualization patterns clear, thus easing the analysis of the honeypot phenomena.

It has been shown how CMLHL provides a helpful technique to visualize backscatter attacks, as well as to identify those attacks that overflow a buffer and download malware.

The neural visualization technique builds on previous works, by providing insight into honeynet data to intuitively check the performance of Snort. From a general perspective, the value of the Snort classification error rate can be seen from the Snort output visualizations.

After gaining a general idea of the dataset structure, an in-depth analysis conducted a comprehensive analysis of each of the points in the groups identified by CMLHL (Figs. 4-6 and 10-12). As a result, the following conclusions can be drawn for each one of the destination ports:

- 8: We are able to identify the type of ICMP packet, by inserting its code into the field destination port. ICMP type 8 corresponds to ICMP echo or ping, used for probing the Internet, looking for victim hosts.

- 22: SSH appears to be traffic flow with many packets coming from one source to one of the honeypots. They correspond to connection attempts by attackers or infected machines.

- 80: HTTP. Attackers try different vulnerabilities against web applications.
- 135: DCE endpoint resolution, used by Microsoft for Remote Procedure Call protocol. It has always been and still is one of the most exploited services by virus and worms.

- 139: NETBIOS Session Service. Plenty of attacks to this Microsoft Windows service were identified.

- 443: HTTP protocol over TLS/SSL connection attempts.

- 445: SMB directly over IP. As most of the traffic in the biggest group identified by CMLHL is aimed at this destination port, we can conclude that this is a widely exploited service.

- 1433: Microsoft-SQL-Server, used by the old SQL Slammer worm.

- 1521: Oracle TNS Listener. It appears that attackers try to connect to the honeypot via Oracle service.

- 2967: Symantec System Center. Vulnerabilities have been found in the Symantec service, which are exploited in the wild.

- 3128: Proxy Server//Reverse WWW Tunnel Backdoor, where the MyDoom worm operates.

- 3389: MS Terminal Services, used for Remote Desktop.

- 4444: This port is a common return port for the rpc dcom.c buffer overflow and for the msblast rpc worm.

- 4899: Remote Administrator default port. There is a known remotely exploitable vulnerability in some radmin server versions that allows code execution.

- 5061: SIP-TLS. Used for VoIP communications.

- 5900: Virtual Network Computer or VNC, used also as a remote desktop solution.

- Port 8080: HTTP Alternate port, also used as an HTTP proxy.

- Port 19765: Used in Kademlia (Bittorrent protocol).

This in-depth analysis remains necessary, in order to further our understanding of some of the visualized attacks, but CMLHL projections appear sufficient for a swift understanding of Internet attacks.

Further work will focus on the application of different projection/visualization models, as well as on visualization using different metrics, instead of the original features of the data.

More analysis can be done with the data, such as visualization of this attack traffic by each of the 
honeynet infrastructure sensors. In this way, one could compare the pattern of attack behavior, distinguishing the Internet space placement. On the other hand, to speed up the data analysis, High Performance and Parallel Computing can be also applied.

Another interesting improvement of CMLHL visualization may be to provide interactive capabilities; a user or analyst could select one or more points from the projections and the system could give details about the data behind them. In a further approach, the system could automatically generate signatures for user selected clusters, suggesting a solution to the large amount of Snort's undetected packets.

Enrichment of the attack dataset may also be a focus of attention. Researchers are correlating network traffic data with attempts to exploit networks collected during simulated vulnerability exploitation. Malware is also obtained for those attacks that aim to spread the infection. All this data requires further in-depth analysis, and neural projection techniques will assist greatly in that task.

These datasets may be shared with any interested researchers upon request, thereby providing a solution to a known issue and allowing other groups to compare their results.

\section{Acknowledgments}

This research has been partially supported through the Regional Government of Gipuzkoa, the Department of Research, Education and Universities of the Basque Government, and the Spanish Ministry of Science and Innovation (MICINN) under projects TIN2010-21272-C02-01 and CIT-0200002009-12 (funded by the European Regional Development Fund). This work was also supported in the framework of the IT4Innovations Centre of Excellence project, reg. no. CZ.1.05/1.1.00/02.0070 supported by the Operational Program 'Research and Development for Innovations' funded through the Structural Funds of the European Union and the state budget of the Czech Republic.

\section{References}

1. J. M. Myerson, Identifying enterprise network vulnerabilities, International Journal of Network Management 12(3) (2002) 135-144.
2. Computer security threat monitoring and surveillance (James P. Anderson Co, 1980).

3. D. E. Denning, An intrusion-detection model, IEEE Transactions on Software Engineering 13(2) (1987) 222-232.

4. T. Chih-Fong, H. Yu-Feng, L. Chia-Ying and L. Wei-Yang, Intrusion detection by machine learning: A review, Expert Systems with Applications 36(10) (2009) 11994-12000.

5. Z. Bankovi, J. M. Moya, Á. Araujo, D. Fraga, J. C. Vallejo and J. M. de Goyeneche, Distributed intrusion detection system for wireless sensor networks based on a reputation system coupled with kernel self-organizing maps, Integrated Computer-Aided Engineering 17(2) (2010) 87-102.

6. C. Otte and C. Störmann, Improving the accuracy of network intrusion detectors by input-dependent stacking, Integrated Computer-Aided Engineering 18(3) (2011) 291-297.

7. R. A. Becker, S. G. Eick and A. R. Wilks, Visualizing network data, IEEE Transactions on Visualization and Computer Graphics 1(1) (1995) 16-28.

8. A. D. D'Amico, J. R. Goodall, D. R. Tesone and J. K. Kopylec, Visual discovery in computer network defense, IEEE Computer Graphics and Applications 27(5) (2007) 20-27.

9. J. R. Goodall, W. G. Lutters, P. Rheingans and A. Komlodi, Focusing on context in network traffic analysis, IEEE Computer Graphics and Applications 26(2) (2006) 72-80.

10. T. Itoh, H. Takakura, A. Sawada and K. Koyamada, Hierarchical visualization of network intrusion detection data, IEEE Computer Graphics and Applications 26(2) (2006) 40-47.

11. Y. Livnat, J. Agutter, S. Moon, R. F. Erbacher and S. Foresti, A visualization paradigm for network intrusion detection, in Sixth Annual IEEE SMC Information Assurance Workshop, 2005. IAW '05, eds. (2005), pp. 92-99.

12. H. Adeli and S. L. Hung, Machine Learning - Neural Networks, Genetic Algorithms and Fuzzy Systems (John Wiley \& Sons, New York, USA, 1995).

13. H. Adeli and X. Jiang, Dynamic fuzzy wavelet neural network model for structural system identification, Journal of Structural Engineering 132(2006) 102111.

14. M. Ahmadlou and H. Adeli, Enhanced probabilistic neural network with local decision circles: A robust classifier, Integrated Computer-Aided Engineering 17(3) (2010) 197-210.

15. M. Oja, G. O. Sperber, J. Blomberg and S. Kaski, Self-organizing map-based discovery and visualization of human endogenous retroviral sequence groups, International Journal of Neural Systems 15(3) (2005) 163-180.

16. N. Lopes and B. Ribeiro, An evaluation of multiple feed-forward networks on graphics processing 
units, International Journal of Neural Systems 21(1) (2010) 31-47.

17. M. L. Borrajo, B. Baruque, E. Corchado, J. Bajo and J. M. Corchado, Hybrid neural intelligent system to predict business failure in smes, International Journal of Neural Systems 21(4) (2011) 277-296.

18. Á. Herrero, E. Corchado, P. Gastaldo and R. Zunino, Neural projection techniques for the visual inspection of network traffic, Neurocomputing 72(16-18) (2009) 3649-3658.

19. Á. Herrero, E. Corchado, M. A. Pellicer and A. Abraham, Movih-ids: A mobile-visualization hybrid intrusion detection system, Neurocomputing 72(13-15) (2009) 2775-2784.

20. E. Corchado and Á. Herrero, Neural visualization of network traffic data for intrusion detection, Applied Soft Computing 11(2) (2011) 2042-2056.

21. E. Corchado, D. MacDonald and C. Fyfe, Maximum and minimum likelihood hebbian learning for exploratory projection pursuit, Data Mining and Knowledge Discovery 8(3) (2004) 203-225.

22. L. Xu, Best harmony, unified rpcl and automated model selection for unsupervised and supervised learning on Gaussian mixtures, three-layer nets and me-rbf-svm models, International Journal of Neural Systems 11(1) (2001) 43-69.

23. K. A. Charles, Decoy systems: A new player in network security and computer incident response, International Journal of Digital Evidence 2(3) (2004).

24. U. Zurutuza, R. Uribeetxeberria and D. Zamboni, A data mining approach for analysis of worm activity through automatic signature generation, in 1st ACM Workshop on AISec, eds. (ACM, 2008), pp. 61-70.

25. F. Le, S. Lee, T. Wong, H. S. Kim and D. Newcomb, Detecting network-wide and router-specific misconfigurations through data mining, IEEE/ACM Transactions on Networking 17(1) (2009) 66-79.

26. N. Provos, A virtual honeypot framework, in 13th USENIX Security Symposium, eds. (2004), pp.

27. P. Baecher, M. Koetter, T. Holz, M. Dornseif and F. Freiling, The nepenthes platform: An efficient approach to collect malware, in 9th International Symposium on Recent Advances in Intrusion Detection (RAID 2006), eds. (Springer Berlin/Heidelberg, 2006), pp. 165-184.

28. D. Moore, C. Shannon, D. J. Brown, G. M. Voelker and S. Savage, Inferring internet denial-of-service activity, ACM Transactions on Computer Systems 24(2) (2006) 115-139.

29. Á. Alonso, S. Porras, E. Ezpeleta, E. Vergara, I. Arenaza, R. Uribeetxeberria, U. Zurutuza, Á. Herrero and E. Corchado, Understanding honeypot data by an unsupervised neural visualization, in Computational intelligence in security for information systems 2010 Springer Berlin/Heidelberg, pp. 151-160.

30. U. Zurutuza, E. Ezpeleta, Á. Herrero and E. Corchado, Visualization of misuse-based intrusion detection: Application to honeynet data, in 6th international conference on soft computing models in industrial and environmental applications, Springer Berlin/Heidelberg, pp. 561-570.

31. Á. Alonso, S. Porras, E. Ezpeleta, E. Vergara, I. Arenaza, R. Uribeetxeberria, U. Zurutuza, Á. Herrero and E. Corchado, On the visualization of honeypot data through projection techniques, in 10th International Conference on Mathematical Methods in Science and Engineering, eds. (2010), pp. 10381049.

32. J. McHugh, A. Christie and J. Allen, Defending yourself: The role of intrusion detection systems, IEEE Software 17(5) (2000) 42-51.

33. L. Khaled, Computer security and intrusion detection, Crossroads 11(1) (2004) 2-2.

34. J. P. Anderson, Computer security threat monitoring and surveillance (Technical Report, 1980).

35. P. G. Neumann and D. B. Parker, A summary of computer misuse techniques, in 12th National Computer Security Conference, eds. (1989), pp. 396-407.

36. A. Sundaram, An introduction to intrusion detection, Crossroads 2(4) (1996) 3-7.

37. P. Laskov, P. Dussel, C. Schafer and K. Rieck, Learning intrusion detection: Supervised or unsupervised?, in 13th International Conference on Image Analysis and Processing (ICIAP 2005), eds. F. Roli and S. Vitulano (Springer, Heidelberg, 2005), pp. 50-57.

38. J. M. Rizza, Computer Network Security (Springer US, 2005).

39. I. Balepin, S. Maltsev, J. Rowe and K. Levitt, Using specification-based intrusion detection for automated response, in Sixth International Symposium on Recent Advances in Intrusion Detection (RAID 2003), eds. (Springer, Heiderlberg, 2003), pp. 136-154.

40. Libpcap, http://www-nrg.ee.lbl.gov/. Last access: $08 / 25 / 11$.

41. S. M. Bellovin, There be dragons, in Third Usenix Security Symposium, Citeseer (1992), pp. 14-16.

42. B. Cheswick, An evening with berferd in which a cracker is lured, endured and studied, eds. (Citeseer, 1990), pp. 1990.

43. Deception toolkit, http://www.all.net/dtk/. Last access:

44. L. Spitzner, Honeypots: Tracking Hackers (AddisonWesley Professional, 2003).

45. L. Spitzner, Honeypots: Sticking to hackers, Network Magazine 18(4) (2003) 48-51.

46. C. Ahlberg and B. Shneiderman, Visual information seeking: Tight coupling of dynamic query filters with starfield displays, in Readings in information visualization: Using vision to think Morgan Kaufmann Publishers Inc. (1999), pp. 244-250.

47. J. R. Goodall, W. G. Lutters, P. Rheingans and A. Komlodi, Preserving the big picture: Visual network traffic analysis with tnv, in IEEE Workshop on 
Visualization for Computer Security (VizSEC 05), eds. (IEEE Computer Society, 2005), pp. 47-54.

48. J. Allen, A. Christie, W. Fithen, J. McHugh, J. Pickel and E. Stoner, State of the practice of intrusion detection technologies (Carnegie Mellon University - Software Engineering Institute 2000).

49. T.-W. Yue and S. Chiang, A neural-network approach for visual cryptography and authorization, International Journal of Neural Systems 14(3) (2004) 175-187.

50. J. Zhou, Q. Z. Xu, W. J. Pei, Z. He and H. Szu, Step to improve neural cryptography against flipping attacks, International Journal of Neural Systems 14(6) (2004) 393-405.

51. T. Goldring, Scatter (and other) plots for visualizing user profiling data and network traffic, in 2004 ACM Workshop on Visualization and Data Mining for Computer Security, eds. (ACM Press, 2004), pp. 119-123.

52. A. Lazarevic, V. Kumar and J. Srivastava, Intrusion detection: A survey, in Managing cyber threats: Issues, approaches, and challenges Springer US (2005), pp. 19-78.

53. J. H. Friedman and J. W. Tukey, A projection pursuit algorithm for exploratory data-analysis, IEEE Transactions on Computers 23(9) (1974) 881-890.

54. K. Pearson, On lines and planes of closest fit to systems of points in space, Philosophical Magazine 2(6) (1901) 559-572.

55. H. Hotelling, Analysis of a complex of statistical variables into principal components, Journal of Education Psychology 24 (1933) 417-444.

56. P. Diaconis and D. Freedman, Asymptotics of graphical projection pursuit, The Annals of Statistics 12(3) (1984) 793-815.

57. E. Corchado and C. Fyfe, Connectionist techniques for the identification and suppression of interfering underlying factors, International Journal of Pattern Recognition and Artificial Intelligence 17(8) (2003) 1447-1466.

58. C. Fyfe and E. Corchado, Maximum likelihood hebbian rules, in 10th European Symposium on Artificial Neural Networks (ESANN 2002), eds. (2002), pp. $143-148$.

59. E. Corchado, Y. Han and C. Fyfe, Structuring global responses of local filters using lateral connections, Journal of Experimental \& Theoretical Artificial Intelligence 15(4) (2003) 473-487.

60. H. S. Seung, N. D. Socci and D. Lee, The rectified gaussian distribution, Advances in Neural Information Processing Systems 10 (1998) 350-356.

61. E. Corchado, P. Burgos, M. D. Rodriguez and V. Tricio, A hierarchical visualization tool to analyse the thermal evolution of construction materials, in CDVE 2004, eds. Y. Luo (Springer, Heidelberg, 2004), pp. 238-245.
62. E. Corchado, M. A. Pellicer and M. L. Borrajo, A mlhl based method to an agent-based architecture, International Journal of Computer Mathematics (Accepted - In press) (2009).

63. Á. Herrero, E. Corchado, L. Sáiz and A. Abraham, Dipkip: A connectionist knowledge management system to identify knowledge deficits in practical cases, Computational Intelligence (Accepted - in press) (2009).

64. P. Demartines and J. Herault, Curvilinear component analysis: A self-organizing neural network for nonlinear mapping of data sets, IEEE Transactions on Neural Networks 8(1) (1997) 148-154.

65. T. Kohonen, The self-organizing map, Proceedings of the IEEE 78(9) (1990) 1464-1480.

66. E. López-Rubio, R. M. Luque-Baena and E. Domínguez, Foreground detection in video sequences with probabilistic self-organizing maps, International Journal of Neural Systems 21(3) (2011) 225246.

67. R. Lippmann, J. W. Haines, D. J. Fried, J. Korba and K. Das, The 1999 darpa off-line intrusion detection evaluation, Computer Networks 34(4) (2000) 579-595.

68. R. P. Lippmann, D. J. Fried, I. Graf, J. W. Haines, K. R. Kendall, D. McClung, D. Weber, S. E. Webster, D. Wyschogrod, R. K. Cunningham and M. A. Zissman, Evaluating intrusion detection systems: The 1998 darpa off-line intrusion detection evaluation, in DARPA Information Survivability Conference and Exposition (DISCEX '00), eds. (2000), pp. 12-26.

69. R. Lippmann, J. W. Haines, D. J. Fried, J. Korba and K. Das, Analysis and results of the 1999 darpa off-line intrusion detection evaluation, in Third International Workshop on the Recent Advances in Intrusion Detection (RAID 2000), eds. H. Debar, L. Mé and S. F. Wu (Springer, Heidelberg, 2000), pp. $162-182$.

70. Kdd cup 1999 dataset, http://archive.ics.uci. edu/ml/databases/kddcup99/kddcup99.html. Last access: 24/06/2009

71. M. Elkan, Results of the kdd'99 classifier learning contest, http://www-cse.ucsd.edu/users/elkan/ clresults.html, 1999 .

72. J. McHugh, Testing intrusion detection systems: A critique of the 1998 and 1999 darpa off-line intrusion detection system evaluation as performed by Lincoln laboratory, ACM Transactions on Information and System Security 3(4) (2000) 262-294.

73. S. Maheshkumar and S. Gursel, Why machine learning algorithms fail in misuse detection on kdd intrusion detection data set, Intelligent Data Analysis 8(4) (2004) 403-415.

74. Euskalert, Basque honeypot network, http://www. euskalert.net. Last access: 10/05/2010. 